\title{
An analysis of polygenic risk scores for non-alcoholic fatty liver disease
}

\author{
Dae Won Jun \\ Department of Internal Medicine, College of Medicine, Hanyang University, Seoul, Korea
}

Keywords: Non-alcoholic fatty liver disease; Polymorphism, Single nucleotide; Genes

\section{See Article on Page 486}

After the human genome sequence was decoded in the early 21st century, high-throughput genomics technology has been growing remarkably. It is now possible to analyze the genotype of hundreds of thousands of single nucleotide polymorphisms (SNPs) at a low cost and in a short time with the advancement of technology in large-capacity SNP microarray chips. A genome-wide association study (GWAS) carried out using the SNP chip, and it suggested genetic biomarkers that affect clinical phenotype based on statistical significance among hundreds of thousands of SNPS present on the chip. However, there are several problems with GWAS analyses. First, approximately $90 \%$ of the genetic variants discovered through a GWAS are located in the non-coding or intergenic regions. Most SNPs are not functional genetic mutations that affect the phenotype of a gene. Second, the effect size (fenestration) of genetic variation discovered through GWAS is usually small. Further, the odds ratio for certain diseases is robust in the disease cohort but not high enough in the general population.

In the current issue of Clinical and Molecular Hepatology, Ting et al.' validated that HSD17B13 (rs72613567 and rs6834314) variants showed an inverse association with non-alcoholic fatty liver disease (NAFLD) in the Chinese population but not in Malays or Indians. The study provided several important insights. First, the HSD17B13 variant also has an inverse relationship with NAFLD in Asians. A more interesting finding is that the effects of HSD17B13 variants were different among Asian ethnicities. Not only the prevalence of SNPs but also the effect size of SNPs differs according to ethnic background. Therefore, it is important to validate the influence of SNPs on ethnic differences. The study showed that the protective effects of HSD17B13 variants in Asians were different from those in Chinese, Malays, and Indians. Second, a GWAS study analyzed hundreds of thousands of genes at the same time, so statistical errors may occur; it may sometimes be difficult to match the control group. Therefore, caution should be exercised when accepting the odds ratio obtained in a cross-sectional study as a risk factor. GWAS methodology needs to be validated before the results of prospective cohort studies are accepted as determining risk factors. Here, the first such longitudinal cohort study performed in Asia is presented.

Some SNPs show high penetration with a $70-80 \%$ probability

\begin{abstract}
Abbreviations:
GRS, genetic risk score; GWAS, genome-wide association study; NAFLD, nonalcoholic fatty liver disease; SNPs, single nucleotide polymorphisms
\end{abstract}

\section{Corresponding author: Dae Won JUN}

Department of Internal Medicine, Hanyang University Hospital, College of Medicine, Hanyang University, 222-1 Wangsimni-ro, Seongdong-gu, Seoul 04763, Korea

Tel: +82-2-2290-8334, Fax: +82-504-159-7224

E-mail: noshin@hanyang.ac.kr

https://orcid.org/0000-0002-2875-6139 
of disease development, but NAFLD does not develop because of just one SNP. Most SNPs associated with metabolic diseases showed low fenestration of approximately 1.5-2.5-fold. Therefore, it has been suggested that a genetic risk score (GRS) should be generated to enhance risk prediction using genetic data. Gellert-Kristensen et al. ${ }^{2}$ proposed a new GRS for NAFLD comprising three common variants (PNPLA3, TM6SF2, and HSD17B13); this GRS predicts a 12-fold higher risk of cirrhosis compare to general population. Koo et al. ${ }^{3}$ suggested a polygenic risk score based on polymorphisms in PNPLA3 and TM6SF2 together with clinical factors that identified steatohepatitis among fatty liver disease. The presence of an SNP variant does not necessarily mean that the disease will develop in near future. Rather, environmental factors such as smoking, lack of exercise, overeating, and alcohol consumption are more important factors that cause NAFLD. Therefore, genetic and environmental risk factors should be properly integrated and taken into consideration when evaluating patients with NAFLD.

\section{Conflicts of Interest}

The author has no conflicts to disclose.

\section{REFERENCES}

1. Ting YW, Kong AS, Zain SM, Chan WK, Tan HL, Mohamed Z, et al. Loss-of-function HSD17B13 variants, non-alcoholic steatohepatitis and adverse liver outcomes: results from a multi-ethnic Asian cohort. Clin Mol Hepatol 2021;27:486-498.

2. Gellert-Kristensen H, Richardson TG, Davey Smith G, Nordestgaard BG, Tybjaerg-Hansen A, Stender S. Combined effect of PNPLA3, TM6SF2, and HSD17B13 variants on risk of cirrhosis and hepatocellular carcinoma in the general population. Hepatology 2020;72:845856.

3. Koo BK, Joo SK, Kim D, Lee S, Bae JM, Park JH, et al. Development and validation of a scoring system, based on genetic and clinical factors, to determine risk of steatohepatitis in Asian patients with nonalcoholic fatty liver disease. Clin Gastroenterol Hepatol 2020;18:2592-2599.e10. 